\title{
Enhancement of Wound Healing by the Traditional Chinese Medicine Herbal Mixture Sophora flavescens in a Rat Model of Perianal Ulceration
}

\author{
XIAOPING XU ${ }^{1}$, XIAOHUA LI ${ }^{1}$, LEI ZHANG ${ }^{1}$, ZHAOHUI LIU ${ }^{1}$, YUAN PAN ${ }^{1}$, DONG CHEN ${ }^{1}$, DONGHUA BIN $^{2}$, \\ QUN DENG ${ }^{3}$, YU SUN ${ }^{4}$, ROBERT M. HOFFMAN ${ }^{5,6}$, ZHIJIAN YANG $^{4,5}$ and HONG YUAN ${ }^{7}$ \\ ${ }^{1}$ Department of Anorectal Surgery, Yuhang District First People's Hospital, Hangzhou, P.R. China; \\ ${ }^{2}$ Department of Anorectal Surgery, Affiliated First Hospital of Hunan \\ University of Traditional Chinese Medicine, Changsha, P.R. China; \\ ${ }^{3}$ Department of Oncological Surgery, Affiliated Second Hospital of Medical \\ College of Zhejiang University, Hangzhou, P.R. China; \\ ${ }^{4}$ Origin Biosciences Inc., Nanjing, P.R. China; \\ ${ }^{5}$ AntiCancer, Inc., San Diego, CA, U.S.A.; \\ ${ }^{6}$ Department of Surgery, University of California San Diego, San Diego, CA, U.S.A.; \\ ${ }^{7}$ Department of Cardiology, Yuhang District First People's Hospital, Hangzhou, P.R. China
}

\begin{abstract}
Background/Aim: Hemorrhoidectomy is often associated with significant postoperative complications that may result in slow wound healing. The traditional Chinese medicine (TCM) compound Sophora flavescens (CSF) has shown efficacy on many inflammatory disorders. The aim of the present study was to examine the efficacy of CSF on wound healing in a rat model of perianal ulceration. Materials and Methods: A rat model of perianal ulceration was induced by subcutaneous injection of $75 \%$ glacial acetic acid. The animals with induced perianal ulcer received topical treatment of low, medium, and high doses of CFS twice daily. Potassium permanganate (PP); 0.02\%) was given to the animals for comparison. Macroscopic and histological assessments of the ulcerated area were performed after treatment. The expression of pro-inflammatory cytokines prostaglandin $E_{2}\left(P G E_{2}\right)$ and interleukin-8 (IL-8) was detected by immunohistochemical analysis. Results: Topical administration of medium- and high-dose CSF significantly enhanced perianal ulcer healing as compared to the
\end{abstract}

This article is freely accessible online.

Correspondence to: Hong Yuan, Department of Cardiology, Yuhang District First People's Hospital, Hangzhou, Zhejiang Province, P.R. China. Tel: +86 15372002098, Fax: +86 057186224254, e-mail: 15799621@qq.com

Key Words: Compound Sophora flavescens, topical treatment, cytokine, perianal ulceration. untreated control $(p<0.05)$. The macroscopic ulceration score was significantly reduced only in the high-dose CSF-treated group as compared to the control $(p<0.01)$. All doses of CSF and PP ameliorated histological damages in the rats with induced perianal ulceration. High-dose CSF or PP significantly reduced the expression of $P G E_{2}$ and $I L-8$ as compared to the control $(p<0.01)$. No treatment-related toxicity was found in either the CSF-or the PP-treated mice. Conclusion: CSF enhances wound healing in a rat model of perianal ulceration. The inhibitory effect of CSF on proinflammatory cytokines $P G E_{2}$ and IL-8 may be involved in the mechanism of enhanced wound-healing.

Hemorrhoidectomy is often associated with significant postoperative complications, including edema, pain, bleeding and ulceration, resulting in a protracted period of wound healing and recovery (1-9). Perianal tissue inflammation around the open wound can occur after hemorrhoidectomy (10, 11). Various types of medication, including metronidazole, glyceryl trinitrate $(0.2 \%)$, steroids, local anesthetics (bupivacaine), anti-inflammatory drugs, hemorrhoid creams, are being used for treatment of complications of hemorrhoidectomy with variable outcome (12-14).

Traditional Chinese medicine (TCM) has a long history of human use in China and other Asian countries for treating and preventing diseases. Compound Sophora flavescens (CSF) is a TCM formula consisting of a combination of 10 herbs, including Sophora flavescens, Phellodendron amurense, Radix sanguisorbae, Scutellaria baicalensis, white peony, Gardenia florida, Betel nut, Rhubarb, Mint and 
A
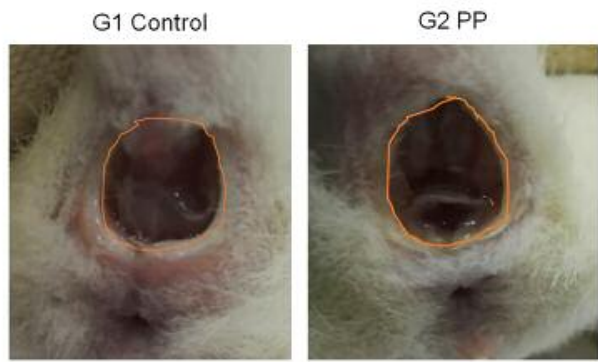

G3 Low dose CSF

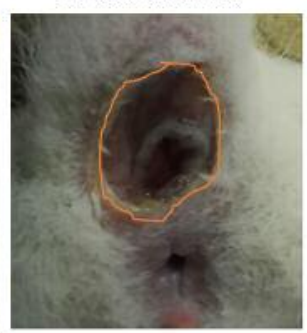

G4 Medium dose CSF

G5 High dose CSF

Day 1
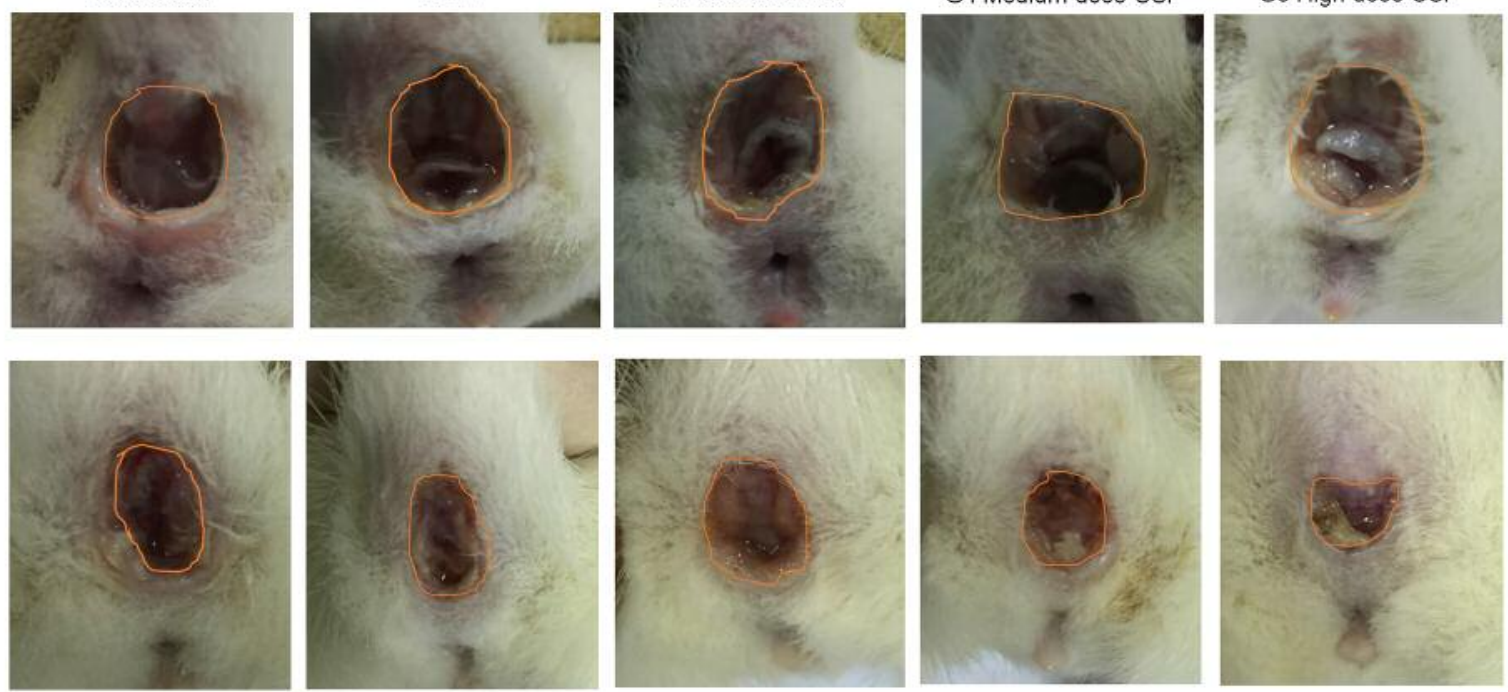

Day 8

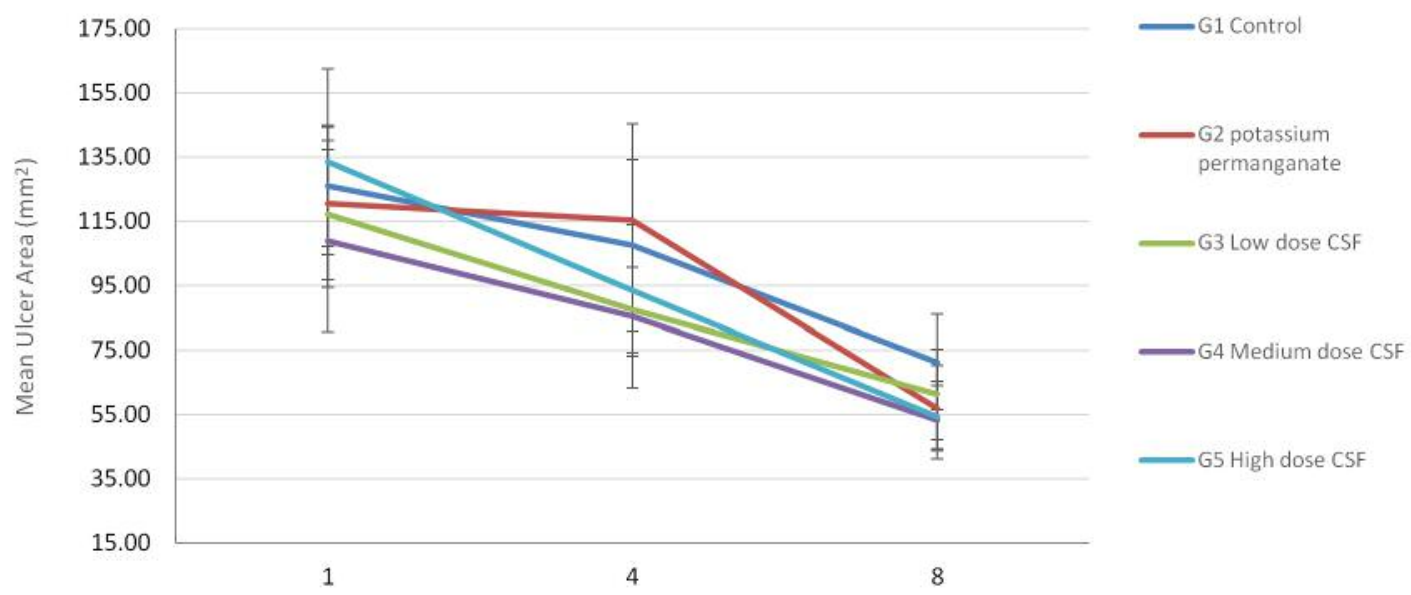

Days after treatment

C

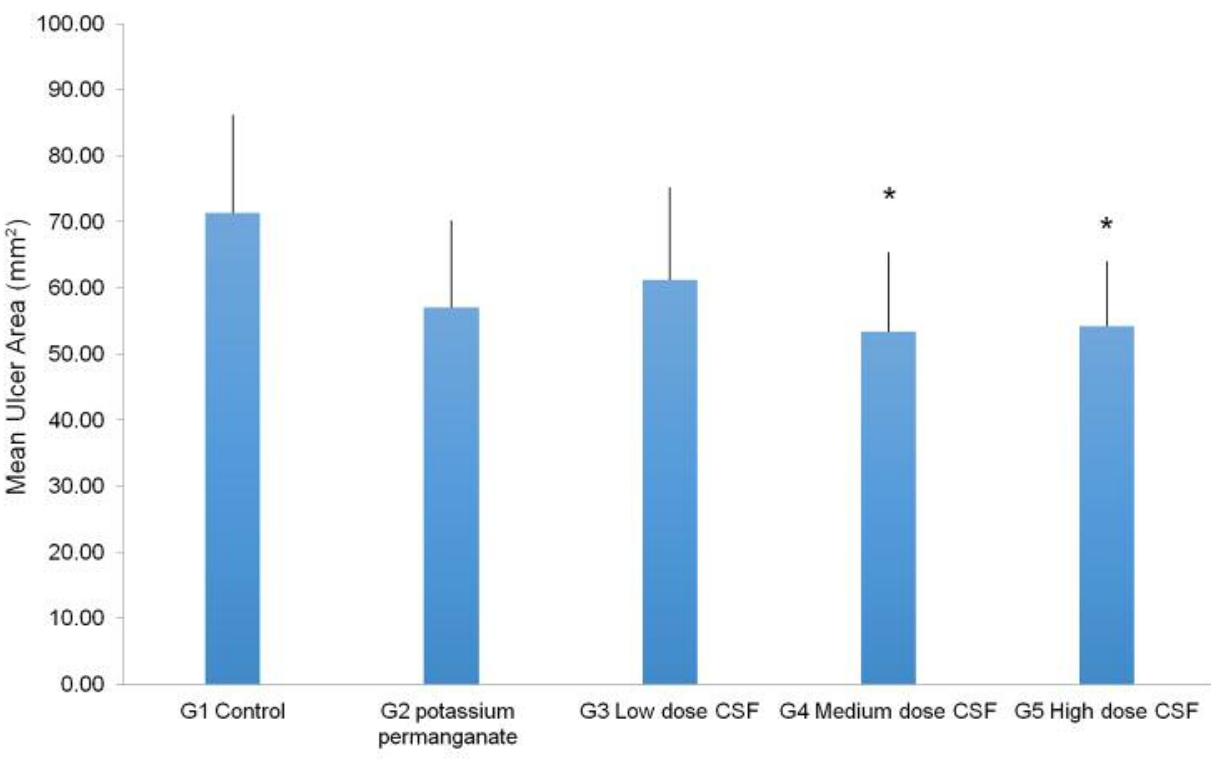

Figure 1. Continued 


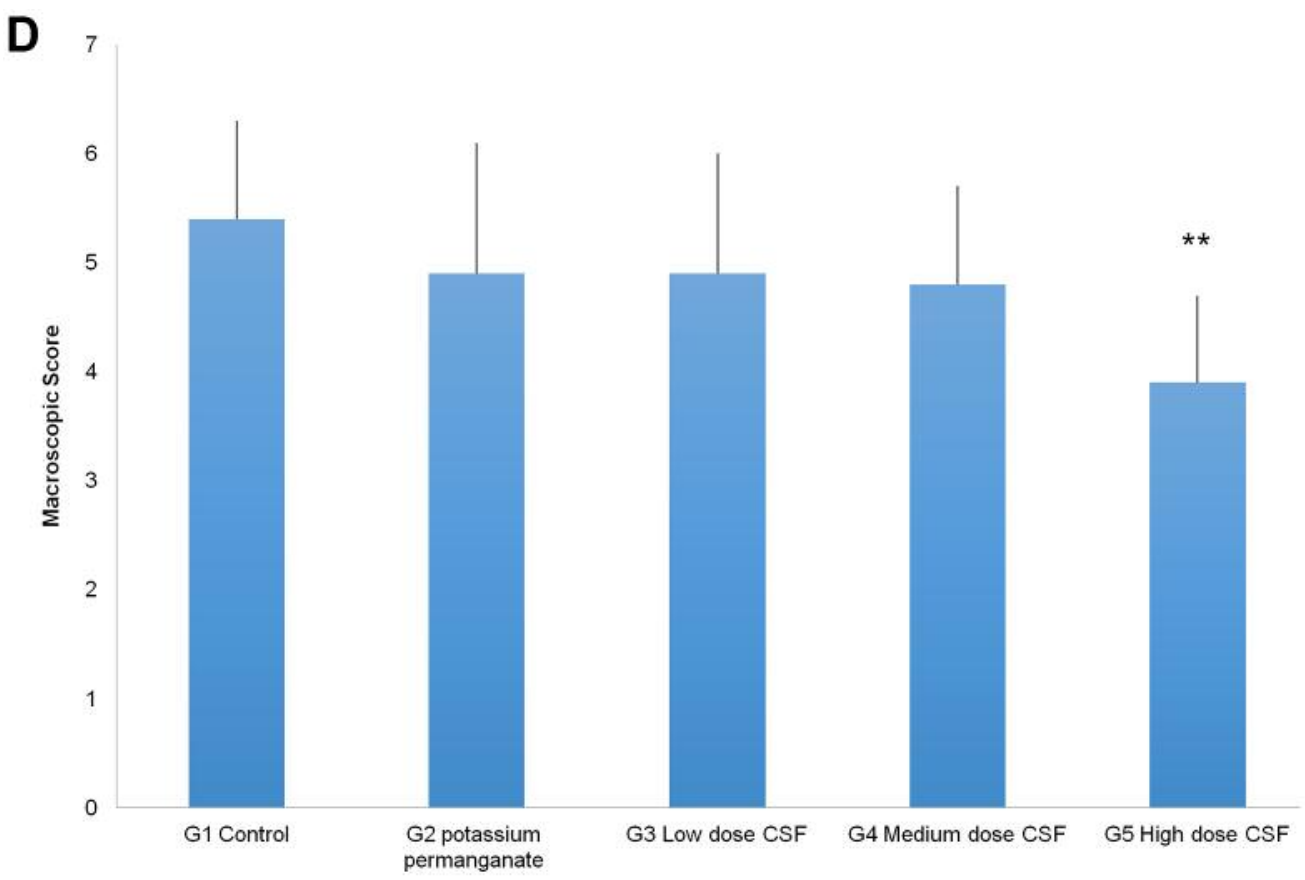

Figure 1. CFS promotes wound healing in the rat model of perianal ulceration. A: Representative images of perianal ulcers in each group (G) before and after treatment. The ulcerated area is outlined. B: Perianal ulceration changes during the course of treatment. C: Mean ulcerated area at the end of treatment. D: Macroscopic pathological scores. Data are represented as mean $\pm S D$ of 8 animals of each group. ${ }^{*} p<0.05$, ${ }^{* *} p<0.01$ when compared to the control group. CFS, Compound Sophora flavescens; PP, potassium permanganate.

Licorice. Sophora flavescens, as a main component, is widely used in traditional herbal medicine for the treatment of fever, inflammatory disorders, ulcers, and skin burns (15). The components in the CSF formula have various effects, including anti-inflammation, antibacterial activity, blood nourishment, pain relief, hemostasis, etc. (16-24). CSF has demonstrated improvement in local microcirculation, control of local inflammation, pain relief, and promotion of wound healing. However, CSF has never been tested for therapy of complications of anal diseases.

In the present study, we established a rat model of perianal ulceration to mimic clinical postoperative complications of anal hemorrhoidectomy and evaluated the efficacy of CSF on wound healing.

\section{Materials and Methods}

Animals. Male Sprague-Dawley (SD) rats (170-210 g) were purchased from the Animal Department of the College of Medicine, Yangzhou University, Yangzhou, P.R China (certificate No. SYXK(Su) 20140012)). Rats were maintained in a high-efficiency particulate arrestance (HEPA)-filtered environment at $24-25^{\circ} \mathrm{C}$ with humidity maintained at 50-60\%. Rats were fed with autoclaved laboratory rodent diet. All animal experiments were approved by the Animal Committee of Nanjing Origin Biosciences, Nanjing, China (OB1409).
Perianal ulceration model. Rats were anaesthetized with $2 \%$ sodium pentobarbital $(0.3 \mathrm{ml} / 100 \mathrm{~g})$ via intraperitoneal injection. The rats were then fixed in a ventral decubitus position. The perianal skin was sterilized with $75 \%$ alcohol. Glacial acetic acid $(40 \mu \mathrm{l}, 75 \%)$ was subcutaneously injected into 4 symmetrical points in the perianal area. The rats were allowed to recover from anesthesia. After 24 hours, the perianal skin developed ulceration with appearance of redness, swelling, and inflammatory exudation.

Treatment. Compound Sophora flavescens lotion (CSF) used in the study was supplied by the Yuhang District First People's Hospital (Hangzhou, Zhejiang Province, P.R. China). Undiluted CSF was used as the high dose. CSF was diluted 2 times with distilled water and used as the medium dose and 3 times diluted as the low dose. All animals with a similar-sized perianal ulceration were randomly assigned to 5 groups of 8 mice each 24 hours after the injection of glacial acetic acid. Group 1 was treated with distilled water twice daily as the negative control; Group 2 was treated with PP solution $(0.02 \%)$ twice daily; Group 3 received low-dose CFS twice daily; Group 4 received medium-dose CFS twice daily; Group 5 received high-dose CFS twice daily. All treatments were carried out via topical application on the ulcer surface and continued daily for 7 days. Animal body weights and clinical signs were recorded daily over the course of the experiments.

Macroscopic assessment. The ulcer was measured with calipers on days 1,4 , and 8 after initial treatment. The ulcerated area was calculated using the formula $(\operatorname{are} a=a \times b)$, where $a$ and $b$ represent 
Gl Control
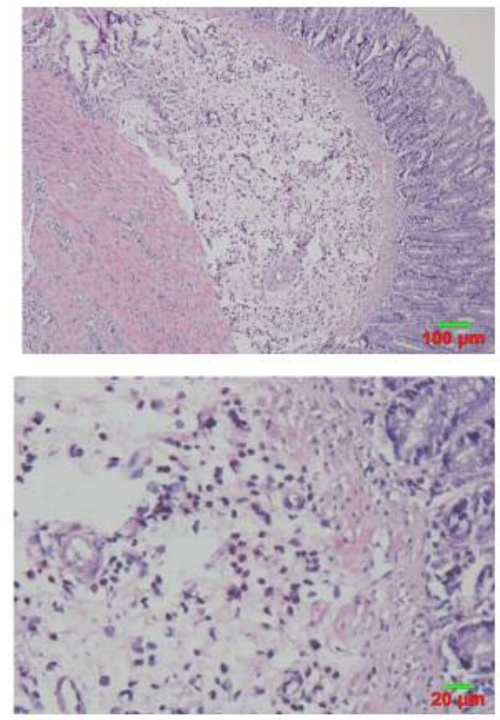

G2 PP
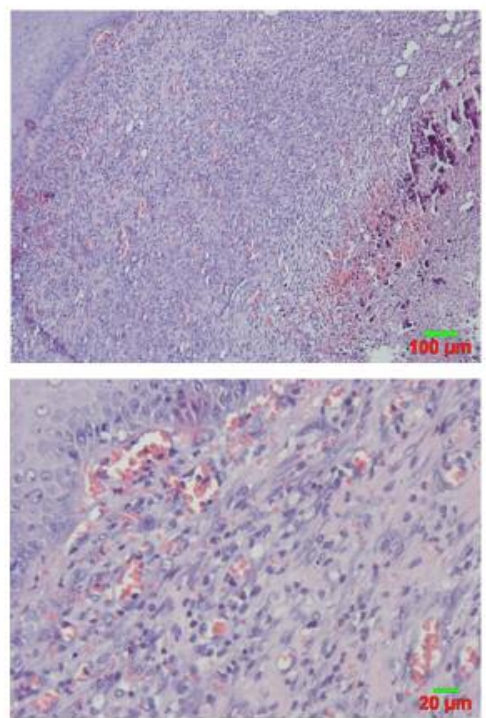

G3 Low dose CSF
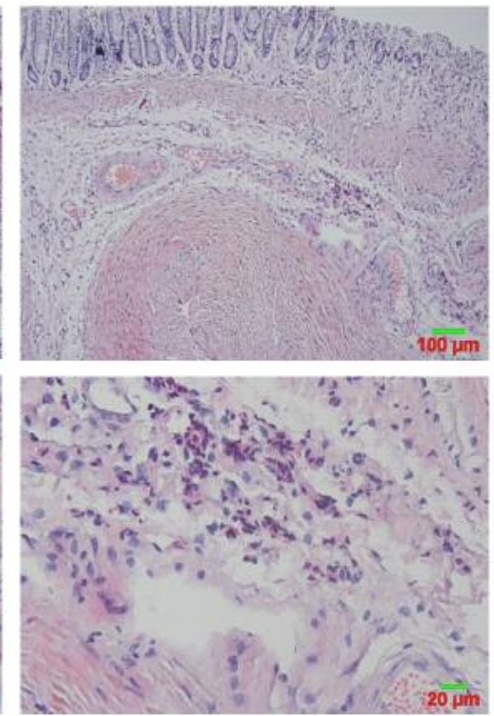

G4 Medium dose CS

G5 High dose CSF
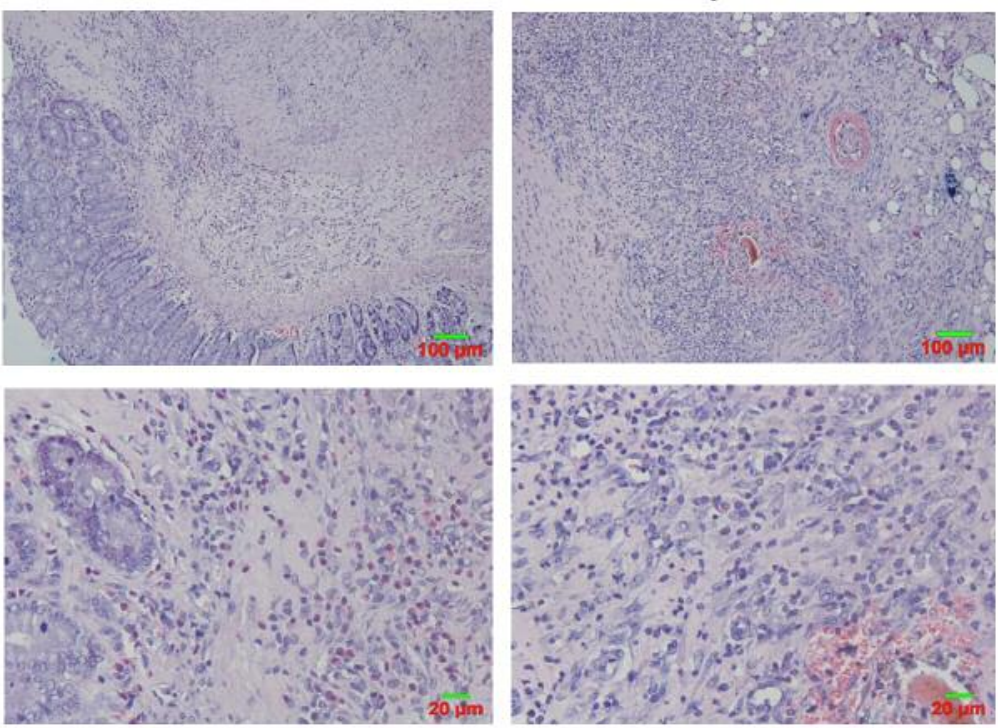

Figure 2. Efficacy of compound Sophora flavescens (CSF) on histological damage in the rat model of perianal ulceration. Representative histological images of hematoxylin and eosin $(H \& E)$ staining of perianal ulcerated tissues in each group $(G)($ magnification: upper panel $\times 100$; lower panel $\times 200)$.

the perpendicular minor dimension and major dimension, respectively. Macroscopic perianal ulceration was assessed using a magnifying glass by an independent observer and scored. The scale for macroscopic damage ranged from $0-6$ based on redness and swelling, severity of inflammatory filtration, and ulcerated area (25). All animals were sacrificed $24 \mathrm{~h}$ after the last treatment. The perianal ulcerated tissues were collected and fixed in $10 \%$ formalin for histological and immunohistochemical analyses.

Histological assessment. For histological examination, the perianal ulcerated tissue was fixed in $10 \%$ formalin, dehydrated, paraffinembedded, sectioned at $4 \mu \mathrm{m}$, and stained with hematoxylin and eosin (H\&E). Histological changes were evaluated by a pathologist who was blinded to the experimental groups.

Immunohistochemical analysis. Paraffin-embedded perianal ulcerated tissue was sectioned at $4 \mu \mathrm{m}$ thickness. All the sections were de-paraffinized and dehydrated through graded ethanol. The sections were washed three times with PBS for $5 \mathrm{~min}$ each, blot dried, and then treated with $3 \%$ hydrogen peroxide for $30 \mathrm{~min}$ at room temperature to block endogenous peroxidase activity. The sections were immersed in antigen-retrieval solution (citrate buffer, $\mathrm{pH}$ 6.0) for $10 \mathrm{~min}$, followed by rinsing with PBS. After blocking with normal goat serum for $30 \mathrm{~min}$ at $37^{\circ} \mathrm{C}$, sections were co- 

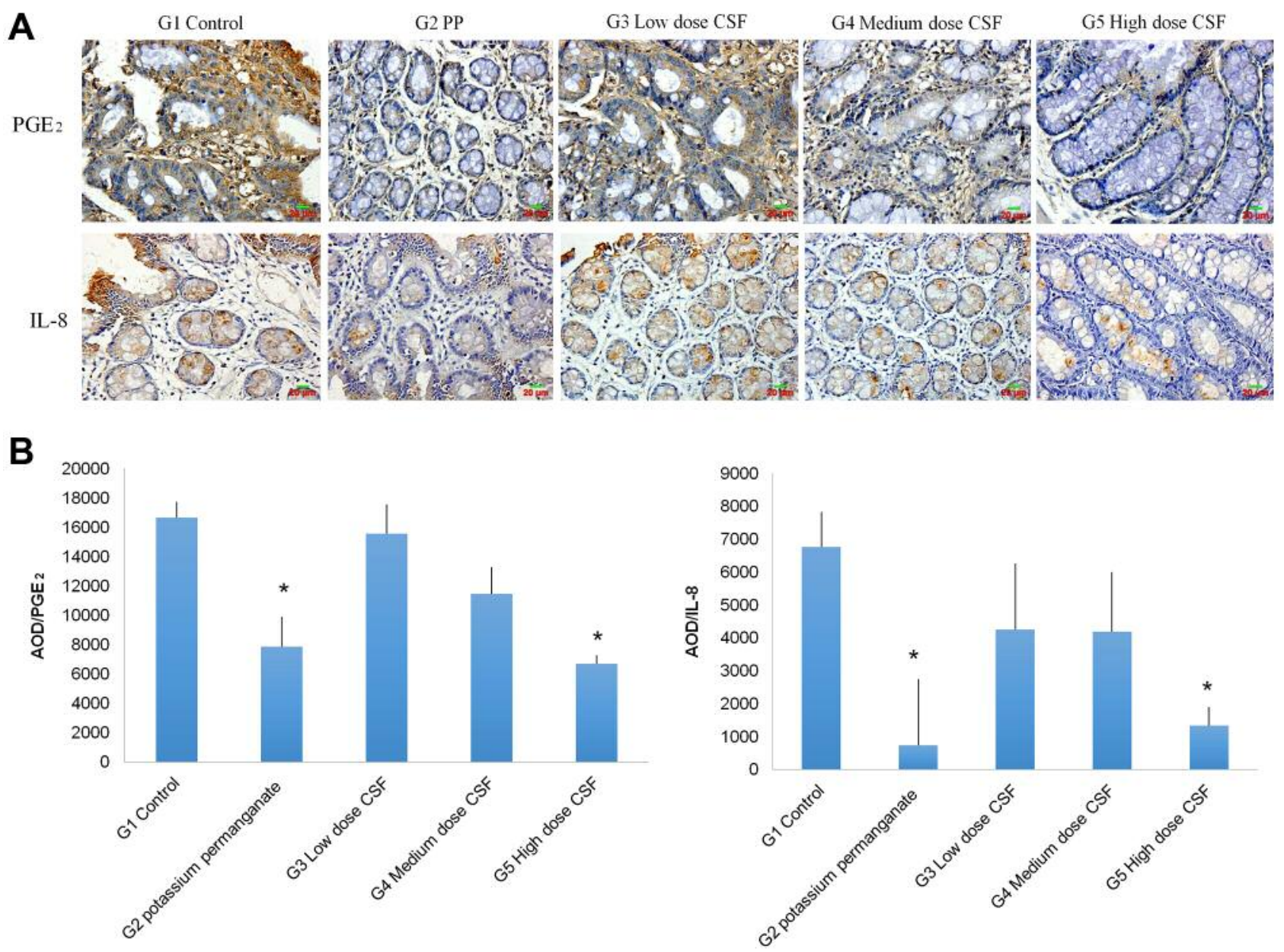

Figure 3. CFS inhibits expression of pro-inflammatory cytokines in the rat model of perianal ulceration. A: Representative images of immunostaining of perianal ulcerated tissues for PGE 2 and IL-8 (magnification, 400X). B: AOD of immunostained perianal ulcerated tissues for PGE $E_{2}$ and IL-8. Data are mean $( \pm S D)$ of 8 animals of each group $(G)$. ${ }^{*} p<0.01$ when compared to the control group. CFS, Compound Sophora flavescens; PP, potassium permanganate; $A O D$, average optical density; $P G E_{2}$, prostaglandin $E_{2} ; I L-8$, interleukin-8.

incubated with primary anti-prostaglandin $\mathrm{E}_{2}\left(\mathrm{PGE}_{2}\right)$ and interleukin-8 (IL-8) antibodies (Biosynthesis Biotechnology Co., Ltd., Beijing, P.R. China) (1:100 dilution in PBS) overnight, and then with a peroxidase-conjugated anti-rabbit $\operatorname{IgG}$ secondary antibody (Biosynthesis Biotechnology Co., Ltd.,) for $1 \mathrm{~h}$ at room temperature. Subsequently, the sections were incubated with 3,3diaminobenzidine (DAB) reagent (ZSGB-BIO, Beijing, P.R.China) for 10 minutes, counterstained with hematoxylin, dehydrated and mounted for microscopy. The slides were viewed at $400 \times$ magnification with positive cells identified by the appearance of brown-stained cells. Expression levels were quantified by the average optical density (AOD) of the positive cells in 5 fields/sample with Image-Pro Plus 6.0 software (Media Cybernetics, Silver Spring, MD, USA).

Statistical analysis. Statistical analysis was performed using SPSS16.0 software (SPSS Inc., Chicago, IL, USA). All results are expressed as mean \pm standard deviation (SD). Comparisons between two or multiple groups were made with the Student's $t$-test or ANOVA. $p<0.05$ was considered significant.

\section{Results and Discussion}

CFS enhances perianal ulcer healing. The rat model of perianal ulceration was developed 24 hours after subcutaneous injection of glacial acetic acid. The rats displayed severe perianal ulceration with redness, swelling, necrosis and inflammation. The efficacy of CSF and PP treatment on the perianal ulcer was evaluated by the measurement of the ulcerated area and macroscopic ulceration score. Representative images and mean ulcerated areas in each treated group are shown in Figures 1A, 1B and $1 \mathrm{C}$. Ulcer healing in the groups treated with medium- and high-dose CSF were enhanced compared with the untreated 


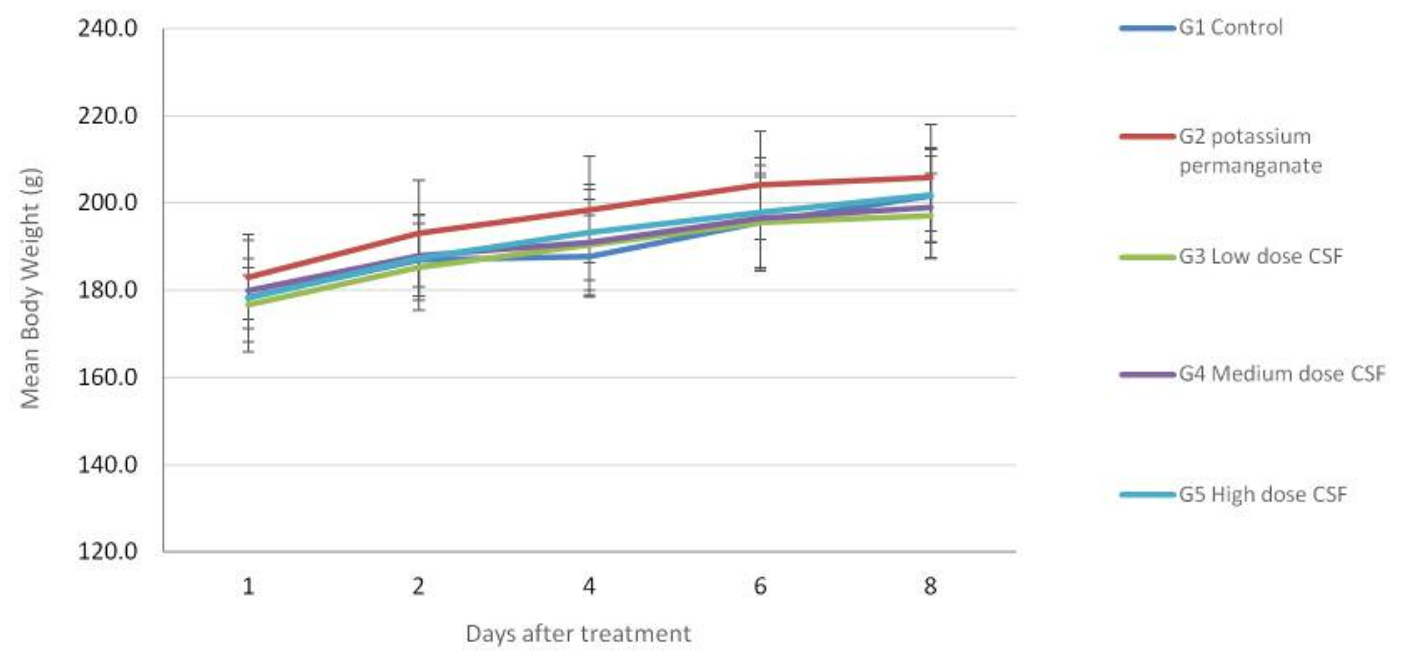

Figure 4. Effect of compound Sophora flavescens (CFS) on body weight in a rat model of perianal ulceration. No significant body weight loss was found in any group of treated rats.

control group $(p<0.05)$. However, the macroscopic ulceration score was only significantly reduced by high-dose CSF compared with the control $(p<0.01)$ (Figure 1D). PP did not significantly reduce the ulcerated area and macroscopic ulceration score as compared with the control $(p>0.05)$. We used PP for comparison, as it is commonly used for postoperative treatment of many perianal diseases.

CFS improves histopathological changes in perianal ulceration. As shown in Figure 2, histologic evaluation of the rats with perianal ulceration showed obvious tissue edema, large squamous epithelium necrosis, bleeding, extravasated blood in mucoderm, numerous neutrophils, and lymphocyte infiltration. These histologic signs were improved in the rats treated with PP and CSF. The high-dose CSF-treated group showed significant reduction of tissue necrosis and inflammatory infiltration as compared with the untreated control group.

CFS inhibits expression of pro-inflammatory cytokines. Proinflammatory cytokines play a very important role in wound healing. The efficacy of CSF on the expression of the proinflammatroy cytokines $\mathrm{PGE}_{2}$ and IL-8 was detected using immunohistochemical analysis. As shown in Figure 3, topical treatment of the perianal ulcer with PP and high-dose CSF resulted in a significant decrease in $\mathrm{PGE}_{2}$ and IL-8 expression in the perianal ulcerated tissue compared to the untreated control group $(p<0.01)$. Our results suggest the reduction of pro-inflammatory cytokines $\mathrm{PGE}_{2}$ and IL-8 is involved in the mechanism of the wound-healing efficacy of CSF.

Efficacy of CFS on body weight and toxicity. Clinical observation and body-weight measurement of animals during the study were performed to assess the toxicity of CFS treatment. No physical or behavioral signs that indicated adverse effects were observed in any treatment group. As shown in Figure 4, a stable body-weight in all treated groups indicated no obvious toxicity.

In conclusion, the present study demonstrates that CSF facilitates wound healing in a rat model of perianal ulceration. The inhibitory effect of CSF on pro-inflammatory cytokines $\mathrm{PGE}_{2}$ and IL- 8 suggests these cytokines may be involved in its wound-healing efficacy mechanism. The present results suggest the potential of the TCM formulation CFS as an effective topical treatment for postoperative complication of hemorrhoidectomy.

\section{Conflicts of Interest}

None of the Authors have a conflict of interest with regard to this study.

\section{Acknowledgements}

This work was supported by the Hangzhou Health Science and Technology Fund No. 201553344.

\section{References}

1 Solorio-López S, Palomares-Chacón UR, Guerrero-Tarín JE, González-Ojeda A, Cortés-Lares JA, Rendón-Félix J, GarcíaRentería J, Chávez-Tostado M, Cuesta-Márquez LA, SalazarParra $M$ and Fuentes Orozco C: Efficacy of metronidazole versus placebo in pain control after hemorrhoidectomy. Results of a controlled clinical trial. Rev Esp Enferm Dig 107: 681-685, 2015. 
2 Shafik A: 'Somatoanal' reflex or 'thermosphincteric' reflex? Dis Colon Rectum 43: 726-728, 2000.

3 Riss S, Weiser FA, Schwameis K, Riss T, Mittlböck M, Steiner $\mathrm{G}$ and Stift A: The prevalence of hemorrhoids in adults. Int $\mathrm{J}$ Colorectal Dis 27: 215-220, 2012.

4 Acheson AG and Scholefield JH: Management of haemorrhoids. BMJ 336: 380-383, 2008.

5 Fox A, Tietze PH and Ramakrishnan K: Anorectal conditions: Hemorrhoids. FP Essent 419: 11-19, 2014.

6 Rivadeneira DE, Steele SR, Ternent C, Chalasani S, Buie WD and Rafferty JL; Standards Practice Task Force of The American Society of Colon and Rectal Surgeons: Practice parameters for the management of hemorrhoids (revised 2010). Dis Colon Rectum 54: 1059-1064, 2011.

7 Wang JY, Lu CY, Tsai HL, Chen FM, Huang CJ, Huang YS, Huang TJ and Hsieh JS: Randomized controlled trial of LigaSure with submucosal dissection versus Ferguson hemorrhoidectomy for prolapsed hemorrhoids. World J Surg 30: 462-466, 2006.

8 Chik B, Law WL and Choi HK: Urinary retention after haemorrhoidectomy: Impact of stapled haemorrhoidectomy. Asian J Surg 29: 233-237, 2006.

9 Pescatori M: Closed vs. open hemorrhoidectomy: Associated sphincterotomy and postoperative bleeding. Dis Colon Rectum 43: 1174-1175, 2000.

10 Pattana-Arun J, Sooriprasoet N, Sahakijrungruang C, Tantiphlachiva $\mathrm{K}$ and Rojanasakul A: Closed vs ligasure hemorrhoidectomy: A prospective, randomized clinical trial. J Med Assoc Thai 89: 453-458, 2006.

11 Sugimoto T, Tsunoda A, Kano N, Kashiwagura Y, Hirose K and Sasaki T: A randomized, prospective, double-blind, placebocontrolled trial of the effect of diltiazem gel on pain after hemorrhoidectomy. World J Surg 37: 2454-2457, 2013.

12 Chung CC, Cheung HY, Chan ES, Kwok SY and Li MK: Stapled hemorrhoidopexy vs. harmonic scalpel hemorrhoidectomy: A randomized trial. Dis Colon Rectum 48: 1213-1219, 2005.

13 Cheetham MJ and Phillips RK: Evidence-based practice in haemorrhoidectomy. Colorectal Dis 3: 126-134, 2001.

14 Carapeti EA, Kamm MA, McDonald PJ, Chadwick SJ and Phillips RK: Randomized trial of open versus closed day-case haemorrhoidectomy. Br J Surg 86: 612-613, 1999.
15 Bae K: Medicinal Plants of Korea. Kyo-Hak Pub, Seoul, p. 261, 2000.

16 Piao XL, Piao XS, Kim SW, Park JH, Kim HY and Cai SQ: Identification and characterization of antioxidants from Sophora flavescens. Biol Pharm Bull 29: 1911-1915, 2006.

17 Kuroyanagi M, Arakawa T, Hirayama Y and Hayashi T: Antibacterial and antiandrogen flavonoids from Sophora flavescens. J Natural Products 62: 595-1599, 1999.

18 Yen $\mathrm{CH}$, ed. The pharmacology of Chinese herbs. 1st ed. Taipei: Chin-Yin Publishing: 376-377, 1999.

19 Yang JH, Hwang YH, Gu MJ, Cho WK and Ma JY: Ethanol extracts of Sanguisorba officinalis L. suppress TNF- $\alpha /$ IFN- $\gamma$ induced pro-inflammatory chemokine production in $\mathrm{HaCaT}$ cells. Phytomedicine 22: 1262, 2015.

20 Dong YC, Shang YZ: Advances of Scutellaria Barbata in pharmacology. J Chengde Med Coll 26: 98-100, 2009.

21 Pharmacopoeia of People's Republic of China (2010) [S]. Beijing: China Medical Science Press: 96-97, 2010.

22 Chu NS: Cardiovascular responses to betel chewing. J Formos Med Assoc 92(9): 835- 837, 1993.

$23 \mathrm{Lu} \mathrm{K}$, Zhang C, Wu W, Zhou M, Tang Y and Peng Y: Rhubarb extract has a protective role against radiation-induced brain injury and neuronal cell apoptosis. Mol Med Rep 2: 2689-2694, 2015.

24 Wang JJ, Chen XQ, Wang W, Zhang Y, Yang ZY, Jin Y, Ge HM, $\mathrm{Li}$ E and Yang G: Glycyrrhizic acid as the antiviral component of Glycyrrhiza uralensis Fisch against coxsackievirus A16 and enterovirus 71 of hand foot and mouth disease. J Ethnopharmacol 147: 114-121, 2013.

$25 \mathrm{Chu}$ QB, Hong S and Zheng Xl: Progress in preparation methodology of animal models of hemorrhoids. Chinese J Comp Med 17: 119-122, 2007.

Received April 13, 2017

Revised May 24, 2017

Accepted May 25, 2017 